\title{
Myocardial Edema Attenuation and Sphingosine- 1-Phosphate
}

\author{
David Straus \\ Rush University Medical Center, Chicago
}

\begin{abstract}
Keywords:
Myocardial

oedema,

Sphingosine-1

Phosphate.
\end{abstract}

\begin{abstract}
:
Introduction: Myocardial edema contributed to cardiac dysfunction in different clinical circumstances. The precise pathophysiology of myocardial edema and therapeutic interventions that target it have remained largely unexplored. The lysophospholipid, Sphingosine-1-Phosphate (S1P) has been shown to decrease edema in the lung through modulation of pulmonary endothelial barrier function. We apply this agent to an isolated rat heart model of ischemia-reperfusion injury and examine its effects on subsequent myocardial edema formation.
\end{abstract}

Methods: 18 isolated male Sprague-Dawley rat hearts were used in this experiment. 3 served as nonischemic controls, 7 served as ischemic controls and 8 served as the intervention group. A 20 minute ischemic period was applied to all groups except the non-ischemic controls. In the intervention group, the rat hearts were given a $30 \mathrm{~mL}$ bolus of $10 \mathrm{nM} \mathrm{S1P}$ prior to ischemia. After completion, heart were histologically analyzed to evaluate the extent of myocardial edema.

Results: In the non-ischemic controls there was $13.65 \%(+/-0.73 \%)$ extracellular area and $84.56 \%(+/$ $0.89 \%)$ intracellular area. In the ischemic controls there was $24.50 \%(+/-3.92 \%)$ extracellular area and $74.11 \%(+/-3.90 \%)$ intracellular area. In the S1P treatment group, there was $21.55 \%(+/-2.6 \%)$ extracellular area and $76.77 \%(+/-2.70 \%)$ intracellular area. These differences did not reach statistical significance $(p>0.05)$.

Conclusion: In this experimental design we observed a non-significant trend in histologic myocardial edema in the S1P treatment group. We also observed a correlated trend in improved myocardial function in the S1P treatment group.

(Cardiovasc. j. 2013; 6(1): 47-51)

\section{Introduction:}

Myocardial edema contributes to cardiac dysfunction and has various iatrogenic and pathologic etiologies. Iatrogenic causes include cardiopulmonary bypass (CPB), cardioplegia and orthotropic heart transplantation. ${ }^{1}$ Pathologic causes include myocardial ischemia/infarction, arterial hypertension, pulmonary hypertension and sepsis. ${ }^{1}$ Preventing fluid collection in the myocardial interstitium is particularly relevant to cardiac surgery since common surgical interventions (CPB and cardioplegia) are major iatrogenic sources of myocardial edema. Compromising cardiac function in this high-risk population may mute the benefits of cardiac surgical interventions by retarding postoperative recovery, increasing post-operative morbidity and increasing post-operative mortality. Defending the myocardial interstitium from edematous fluid overload offers a means to preserve cardiac function from various pathophysiological and iatrogenic insults.

The precise pathophysiological mechanisms of myocardial edema-induced cardiac dysfunction remain poorly defined. However, numerous studies using various models have demonstrated both systolic and diastolic dysfunction linked to myocardial edema. Preload recruitable stroke work (PRSW), an index of myocardial contractility independant of preload and afterload, is found to be decreased in edematous hearts. This decrease in PRSW responds in a dose-dependant fashion to increases in myocardial water content. ${ }^{2}$ Diastolic dysfunction in myocardial edema is seen as an increase in the isovolumic relaxation time constant (ô $)^{2}$ and an increase in myocardial stiffness (i.e. a decrease in compliance effecting both the active and passive phases of ventricular relaxation. These combined effects result in decreased

Address of Correspondence : David Straus, Rush University Medical Center, Department of Neurological Surgery, Chicago, IL, USA. Email: david_straus@rush.edu 
cardiac output (CO): a $3.5 \%$ increase in myocardial water content decreases $\mathrm{CO}$ by $40 \%$ at a given preload. ${ }^{3}$ Decreased ventricular compliance and increased water content may also reduce cardiac efficiency as myocytes are forced to contract against increased intra- myocardial/ interstitial pressures and as the mass (inertia) of the myocardium increases with increasing water content. Ultimately, myocardial edema increases cardiac energy requirements. ${ }^{4}$ Moreover, edema may lead to myocardial ischemia as diffusion distances increase with expanding interstitial space ${ }^{4}$ and as coronary vascular resistance is increased ${ }^{5}$ with elevated interstitial pressures and the resulting capillary bed compression. Finally, myocardial edema may trigger interstitial cardiac fibrosis, ${ }^{3}$ thus chronically impeding cardiac function.

Sphingosine-1-phosphate, a lysophospholipid acting at various endothelial differentiation gene (EDG) receptors, has been shown to increase endothelial barrier function in human pulmonary artery endothelial cells, reducing both inflammation, microvascular leak and ultimately producing a protective effect against pulmonary edema formation. ${ }^{6}$ The increase in endothelial cell barrier function is mediated by the EDG1 (S1PR1) receptor. This $\mathrm{S} 1 \mathrm{P}$ receptor induces cell-to-cell adherens junction formation, cell-toextracellular matrix focal adhesion formation and a resultant decrease in vascular permeability. ${ }^{6}$ Since the EDG1 receptor is abundantly expressed in cardiac endothelial cells ${ }^{7}$ we hypothesized that $\mathrm{S} 1 \mathrm{P}$ would, similarly, be protective against edema formation in the myocardium.

\section{Study Methods}

\section{Animals, Groups and Heart Harvest}

Male Sprague-Dawley rats weighing 350-450 grams (Harlan, Indianapolis, IN) were housed in facilities at the University of Chicago until experimentation. Animals were randomized to control or treatment groups by coin toss.

Three experimental groups were used. One group served as a non-ischemic control (Control, $\mathrm{n}=3$ ), another served as an ischemia-reperfusion control (IR, $\mathrm{n}=7)$ and a final group served as the treatment group (S1P, $\mathrm{n}=8)$.
Rats are anesthetized using sodium pentobarbital (30mg bolus). Rats were anesthetized, intubated through a tracheostomy and then heparinized (500 IU bolus) via the IVC. 3 minutes after the heparin bolus the heart is removed from the chest through a sternotomy incision and placed in cold KrebsHenseleit buffer.

\section{Isolated Heart Prep and Working Heart Model}

The aorta was cannulated and the hearts were perfused for 5 minutes in retrograde fashion (Langendorff prep) with a modified KrebsHenseleit solution $\left(37^{\circ} \mathrm{C}, \mathrm{pH} 7.4\right)$. The hearts were switched to antegrade flow (working heart prep) and perfused with modified KrebsHenseleit solution in a closed recirculating system at $37{ }^{\circ} \mathrm{C}$. Fresh perfusate was given every 15 minutes. In the working heart prep, perfusate entered the cannulated left atrium at a pressure of $8 \mathrm{mmHg}$ (preload), and passed into the left ventricle (LV) from which it was spontaneously ejected through the aortic cannula against a pressure of $80 \mathrm{mmHg}$ (afterload) (Figure 1). After an equilibration period of $5 \mathrm{~min}$, hearts were electrically paced at 290-320 BPM via the metal cannula in the left atrium (LA). Heart rate and left atrial, aortic systolic and aortic diastolic pressures were recorded. Cardiac output (flow into the LA) and aortic flow were also measured. Coronary flow was calculated at the difference between cardiac output and aortic flow.

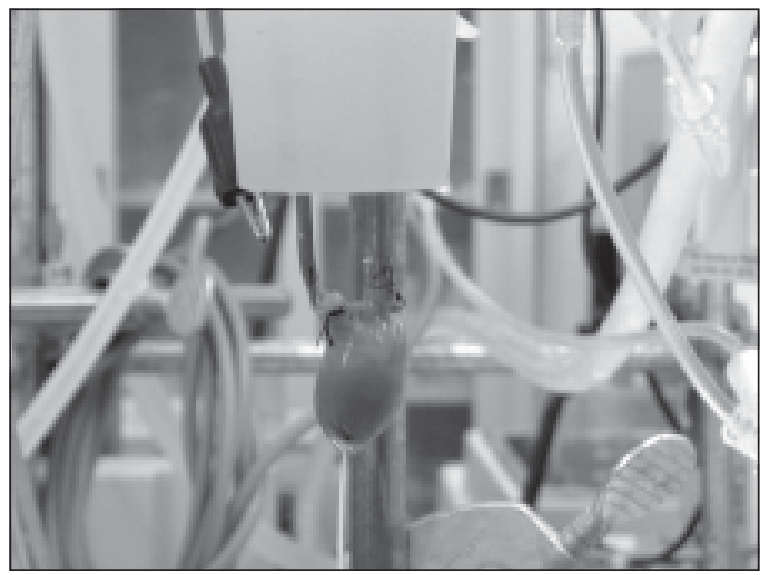

Fig.-1: Isolated working rat heart model demonstrating cannulation of left atrium and aorta. 


\section{Experimental protocol}

Control hearts were mounted on the perfusion apparatus in a Langendorff prep for a 10 minute initiation period. After 10 minutes in the Langendorff prep these hearts were switched into the working heart prep for the remainder of the experiment. IR hearts were mounted on the perfusion apparatus in the Langendorff prep for 10 minutes and were subsequently switched into the working heart prep, as with the Control group. After a 15 minute stabilization period in the working heart prep, however, the IR hearts received a retrograde $30 \mathrm{~mL}$ bolus of perfusate over 3 minutes and were then subjected to global, no-flow ischemia for 20 minutes. Following the ischemic period, IR hearts were reperfused in the Langendorff prep for 5 minutes and then switched back into the working heart prep for the remainder of the experiment. S1P hearts followed a protocol identical to the IR hearts, however the $30 \mathrm{~mL}$ bolus of perfusate administered to the $\mathrm{S} 1 \mathrm{P}$ hearts prior to ischemia contained 10nM S1P (Sigma Chemical, St. Louis, $M O)$. All experiments ended at systolic failure, defined as the loss of antegrade aortic flow. In the working heart prep hearts were paced at 290-320 BPM via the left atrium.

\section{Quantification of Myocardial Edema}

LV tissue samples were stained with H\&E. The ratio of intracellular area to total area and the ratio of interstitial area to total area were measured using Chromavision ACIS software (Clarient, Aliso Viejo, CA).

\section{Statistics}

Data are presented as mean (SEM). Error bars on graphs indicate SEM. Either one-way or twoway ANOVA was used to compare differences among groups when appropriate. Student's $t$ test was used to compare two groups. Significance was taken at $p<0.05$.

\section{Results}

\section{Edema}

Histological analysis of cardiac tissue showed increased extracellular area in the IR group compared to the Control group $(\mathrm{p}=0.12)$ and increased extracellular area in the $\mathrm{S} 1 \mathrm{P}$ group compared to the Control group $(\mathrm{p}=0.12)$.
Additionally, the IR group had decreased intracellular area compared to the Control group $(\mathrm{p}=0.13)$, as did the $\mathrm{S} 1 \mathrm{P}$ group $(\mathrm{p}=0.14)$. In relation to the IR group, the S1P group showed non-significant decreases in extracellular area $(p>0.05)$ and increases intracellular area $(p>0.05)$.

\section{Table-I}

Histological measurements. Data are presented as a percentage of the total area ( \pm SEM).

\begin{tabular}{lcc}
\hline & \% Extracellular Area & \% Intracellular Area \\
\hline Control & $13.65 \%( \pm 0.73 \%)$ & $84.56 \%( \pm 0.89 \%)$ \\
IR & $24.50 \%( \pm 3.92 \%)$ & $74.11 \%( \pm 3.90 \%)$ \\
S1P & $21.55 \%( \pm 2.6 \%)$ & $76.77 \%( \pm 2.70 \%)$ \\
\hline
\end{tabular}

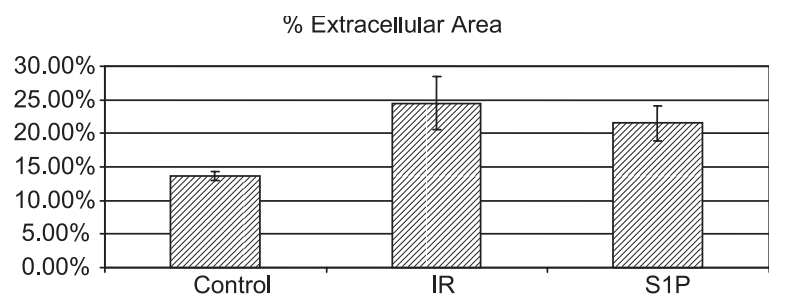

Fig.-2: Extracellular area as a percentage of total area. Error bars represent SEM.

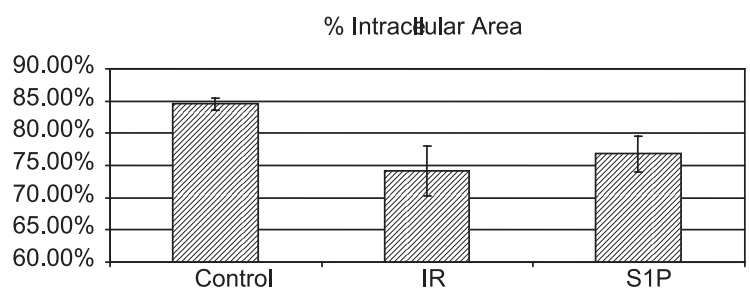

Fig.-3: Intracellular are as a percentage of total area.

\section{Hemodynamics}

Hemodynamic data were recorded every 15 minutes throughout the experiment to monitor cardiac function. The Control group remained hemodynamically stable until the 105 min timepoint, at which point their function steadily declined until failure. Neither the IR group nor the S1P group stabilized hemodynamically. They both demonstrated a post-ischemic loss of systemic flow and a hyperemic increase in coronary flow within the first 15 minutes of reperfusion. These effects offset each other to 
produce a less pronounced decrease in postischemic cardiac output. The hyperemic response seen in the early reperfusion period abated within 30 minutes post-ischemia and coronary flow remained stable until 105 minutes post-ischemia. Functional recovery in both IR and S1P groups peaked at 45 minutes post-ischemia, followed by a steady decline in function until failure.
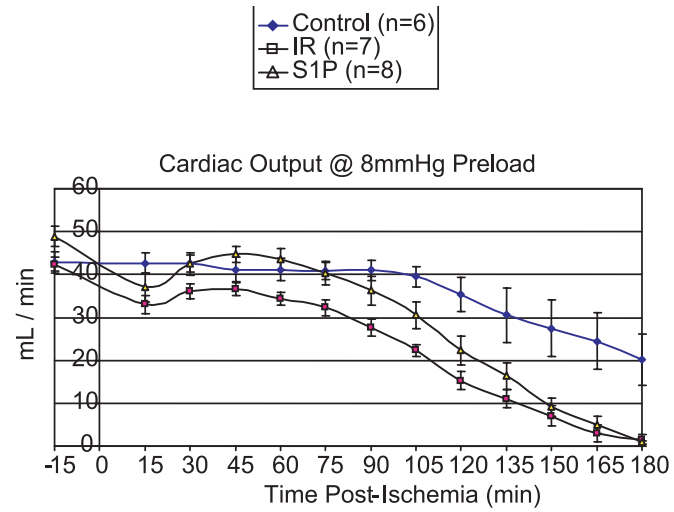

Fig.-4: Cardiac output, calculated as flow through the left atrial cannula.

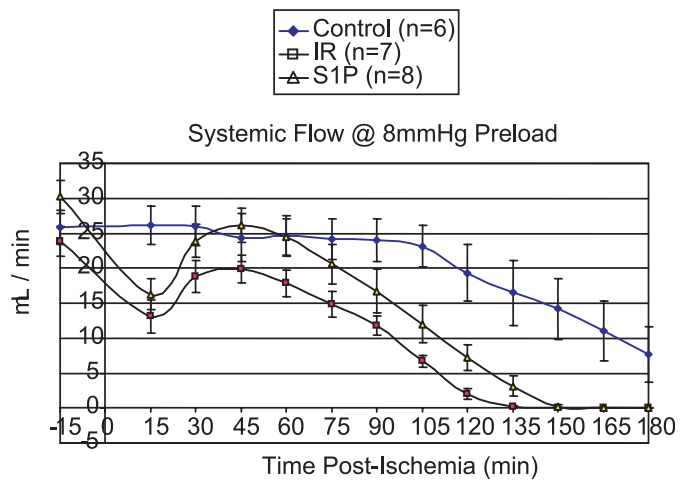

Fig.-5: Systemic flow, calculated as flow through the aorta.

$\rightarrow-$ Control $(n=6)$
$\square-\operatorname{IR}(n=7)$
$\triangle \operatorname{S1P}(n=8)$

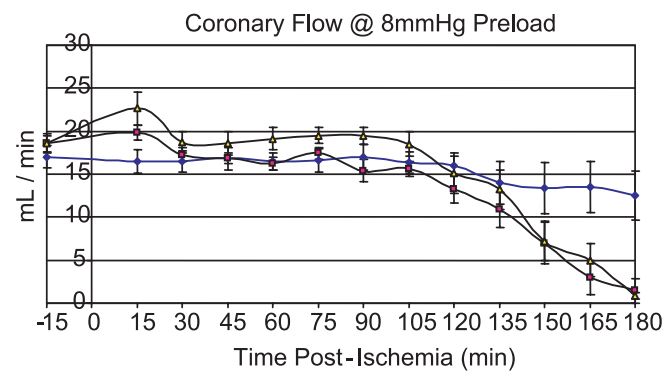

Fig.-6: Coronary flow, calculated as the difference between cardiac output and systemic flow.

\section{Discussion:}

Our data show indications that $\mathrm{S} 1 \mathrm{P}$ may act to decrease interstitial edema in cardiac tissue following ischemia-reperfusion injury. Increases in extracellular area indicate fluid collection within the interstitium. As the interstitial space expands, intracellular area (as a percentage of total area) decreases. Both of these effects are seen in the S1P group and the IR group. Moreover, these effects are more severe in the IR group compared to the $\mathrm{S} 1 \mathrm{P}$ group. These measures show a consistent, albeit nonsignificant, tendency for $\mathrm{S} 1 \mathrm{P}$ treated hearts to recover towards Control values. Moreover, the higher post-ischemic coronary flows (both during the hyperemic recovery period and throughout the experiment) in the $\mathrm{S} 1 \mathrm{P}$ hearts compared to IR hearts may be an additional indicator of decreased edema in S1P treated hearts. As fluid collects in the myocardium, interstitial pressures rise causing an increase in vascular resistance (increased interstitial pressure compresses the microvasculature). Despite trends towards improved functionality of $\mathrm{S} 1 \mathrm{P}$ treated hearts, definitive conclusions about functional differences are hindered by the differing baseline characteristics of the S1P and IR groups. Although coronary flows were nearly identical at baseline, S1P hearts had noticeably higher systemic flows (and, consequently, higher cardiac outputs) at baseline.

This study gives some indication that $\mathrm{S} 1 \mathrm{P}$ may be acting to reduce post-ischemic edema formation in the heart. Despite the observed trends, significance was not obtained in our primary measures of edema and our hemodynamic data is confounded by baseline differences in systemic flows between $\mathrm{S} 1 \mathrm{P}$ and IR hearts. While these experiments may have picked up on some faint signals of edema attenuation, more sensitive methods will be required to further clarify the role of $\mathrm{S} 1 \mathrm{P}$ in myocardial edema.

Currently, we have altered some of our methods in order to re-examine our hypothesis. To measure microvascular permeability directly, we will use Evans-Blue dyed albumin to quantify colloid protein extravasation. We will also add albumin to our modified krebs-henseleit solution 
in order to avoid the use of a crystalloid perfusate. This should serve as a more sensitive marker of edema. Although sustained recovery of systemic flow is the most clinically relevant parameter, we will include hemodynamic measures that are more sensitive to myocardial edema (e.g. PRSW, $\mathrm{dP} / \mathrm{dt}$, tau, $-\mathrm{dP} / \mathrm{dt}$ and LVEDP). Finally, instead of ending the experiments at systolic failure, we will use a standard endpoint relative to the end of ischemia, where functional differences are more pronounced.

\section{References:}

1. Mehlhorn U, Geissler HJ, Laine GA, Allen SJ. Myocardial fluid balance. Eur J Cardio-Thorac 2001;20:1220-1230.

2. Pratt J, Schertel E, Schaefer S, et al. Acute transient coronary sinus hypertension impairs left ventricular function and induces myocardial edema. Am J Physiol Heart C Physiol 1996;271:H834-H841.

3. Laine G, Allen S. Left ventricular myocardial edema. Lymph flow, interstitial fibrosis, and cardiac function. Circ Res 1991;68:1713-1721.

4. Kahles H, Mezger V-A, Hellige G, Spieckermann P, Bretschneider H. The influence of myocardial edema formation on the energy consumption of the heart during aerobiosis and hypoxia. Basic Res Cardiol 1982;77:158-169.

5. Ziegler WH, Goresky CA. Transcapillary Exchange En the Working Left Ventricle of the Dog. Circ Res 1971;29:181207.

6. McVerry BJ, Garcia JG. In vitro and in vivo modulation of vascular barrier integrity by sphingosine 1-phosphate: mechanistic insights. Cell Signal 2005;17:131-139.

7. Mazurais D, Robert P, Gout B, Berrebi-Bertrand I, Laville MP, Calmels T. Cell type-specific localization of human cardiac S1P receptors. J Histochem Cytochem 2002;50:661669.

8. Bianchi C, Araujo EG, Sato K, Sellke FW. Biochemical and structural evidence for pig myocardium adherens junction disruption by cardiopulmonary bypass. Circulation 2001;104:I 319 - I 324.

9. Fallen EL, Elliott W, Gorlin R. Apparatus for study of ventricular function and metabolism in the isolated perfused rat heart. J Appl Physiol 1967;22:836-839.

10. Fischer UM, Cox Jr CS, Stewart RH, Laine GA, Allen SJ. Impact of acute myocardial edema on left ventricular function. J Invest Surg 2006;19:31-38.

11. Gräler MH, Goetzl EJ. The immunosuppressant FTY720 down-regulates sphingosine 1-phosphate G-protein-coupled receptors. FASEB J 2004;18:551-553.
12. Hale JJ, Doherty G, Toth L, et al. Selecting against $\mathrm{S} 1 \mathrm{P}<$ sub $>3</$ sub $>$ enhances the acute cardiovascular tolerability of 3-(N-benzyl) aminopropylphosphonic acid S1P receptor agonists. Bioorg Med Chem lett 2004;14: 3501-3505.

13. Hla T. Physiological and pathological actions of sphingosine 1-phosphate. In: Seminars in cell \& developmental biology. Elsevier, 2004: 513-520.

14. Hoffman Jr JW, Gilbert TB, Poston RS, Silldorff EP. Myocardial reperfusion injury: etiology, mechanisms, and therapies. J Extra Corp Technol 2004;36:391-411.

15. Jin Z-Q, Goetzl EJ, Karliner JS. Sphingosine kinase activation mediates ischemic preconditioning in murine heart. Circulation 2004;110:1980-1989.

16. Jin Z-Q, Zhou H-Z, Zhu P, et al. Cardioprotection mediated by sphingosine-1-phosphate and ganglioside GM-1 in wildtype and PKCå knockout mouse hearts. Am J Physiol - H C Physiol 2002;282:H1970-H1977.

17. Karliner JS. Lysophospholipids and the cardiovascular system. Biochimica et Biophysica Acta BBA-Mol Cell Biol L 2002;1582:216-221.

18. Karliner JS, Honbo N, Summers K, Gray MO, Goetzl EJ. The lysophospholipids sphingosine-1-phosphate and lysophosphatidic acid enhance survival during hypoxia in neonatal rat cardiac myocytes. J Mol Cell Cardiol 2001;33:1713.

19. Nakajima N, Cavalli AL, Biral D, et al. Expression and characterization of Edg 1 receptors in rat cardiomyocytes. Eur J Biochem 2001;267:5679-5686.

20. Ohmori T, Yatomi Y, Osada M, et al. Sphingosine 1phosphate induces contraction of coronary artery smooth muscle cells via S1P2. Cardiovasc Res 2003;58:170-177.

21. Pyne S, Pyne N. Sphingosine 1-phosphate signalling via the endothelial differentiation gene family of G-proteincoupled receptors. Pharmacol Therapeut 2000;88:115-131.

22. Robert P, Tsui P, Laville MP, et al. EDG1 receptor stimulation leads to cardiac hypertrophy in rat neonatal myocytes. J Mol Cell Cardiol2001;33:1589-1606.

23. Saba JD, Hla T. Point-counterpoint of sphingosine 1phosphate metabolism. Circ Res 2004;94:724-734.

24. Sanna MG, Liao J, Jo E, et al. Sphingosine 1-phosphate (S1P) receptor subtypes $\mathrm{S} 1 \mathrm{P} 1$ and $\mathrm{S} 1 \mathrm{P} 3$, respectively, regulate lymphocyte recirculation and heart rate. $J$ Biol Chem 2004;279:13839-48.

25. Schaphorst KL, Chiang E, Jacobs KN, et al. Role of sphingosine-1 phosphate in the enhancement of endothelial barrier integrity by platelet-released products. Am $J$ Physiol Lung Cell Mol Physiol 2003;285:L258-L67. 